\title{
Utility of the Robson Ten Group Classification System to determine appropriateness of caesarean section at a rural regional hospital in KwaZulu-Natal, South Africa
}

\author{
V Makhanya, ${ }^{1}$ MB ChB; L Govender, ${ }^{1}$ MB ChB, FCOG; J Moodley, ${ }^{1,2}$ MB ChB, FRCOG, FCOG, MD \\ ${ }^{1}$ Department of Obstetrics and Gynaecology, Nelson Mandela School of Medicine, College of Health Sciences, University of KwaZulu-Natal, \\ Durban, South Africa \\ ${ }^{2}$ Women's Health and HIV Research Group, Department of Obstetrics and Gynaecology, Nelson Mandela School of Medicine, College of Health \\ Sciences, University of KwaZulu-Natal, Durban, South Africa
}

Corresponding author: V Makhanya (vuyo.makhanya@ymail.com)

Background. High caesarean section (CS) rates are not only costly but associated with significant perinatal and maternal morbidity and mortality. It has recently been suggested that structured auditing of CSs may identify those groups in the obstetric population that contribute substantially to the high rates and for which focused interventions may bring about change.

Objective. To evaluate the utility of the Robson Ten Group Classification System (RTGCS) in determining appropriateness of CS at a regional rural hospital in KwaZulu-Natal Province, South Africa.

Methods. A retrospective review of the hospital records of women delivered by CS over a 3-month period was performed. The RTGCS was used to categorise women according to parity, age, past obstetric history, singleton or multiple pregnancy, fetal presentation, gestational age and mode of onset of labour/delivery.

Results. There were 2553 hospital births over the 3-month study period. The CS rate was $42.4 \%$ (1 082/2 553). According to the RTGCS, groups $1(n=296,27.4 \%), 5(n=186,17.2 \%)$ and $10(n=253,23.4 \%)$ were substantial contributors to the overall CS rate. The main indications for CS were fetal distress (36.5\%) and cephalopelvic disproportion (26.8\%).

Conclusion. The RTGCS is a useful tool with which to identify patient groups warranting interventions to reduce high CS rates in a rural regional hospital setting. Group 1 (nullipara: single cephalic term pregnancy; spontaneous labour) warrants the most attention. Applying stricter criteria and due diligence in decision-making for primary CS may decrease the high CS rates.

S Afr Med J 2015;105(4):292-295. DOI:10.7196/SAMJ.9405

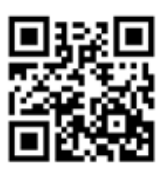

Caesarean section (CS) rates continue to increase globally. The current CS rate at our institution (Lower Umfolozi War Memorial District Hospital, KwaZulu-Natal Province (KZN), South Africa $(\mathrm{SA})$ ) in a low- and middle-income country (LMIC) approaches 50\% (institutional statistics) and has been increasing steadily over time. There is concern about increasing numbers of women with a history of previous CS undergoing CS with subsequent pregnancies. Reducing the CS rate would decrease maternal morbidity without affecting perinatal mortality rates.

Marked differences in CS rates between district, regional and tertiary public sector hospitals in SA have been documented, largely reflecting the effect of high-risk pregnancies on the rate. ${ }^{[1]}$ In a tertiary hospital in Durban, CS rates of $>30 \%$ have been reported, the explanation being the large numbers of highrisk patients. ${ }^{[2]}$

The World Health Organization (WHO) states that CS rates should be between $10 \%$ and $15 \% \cdot{ }^{[3]}$ It has been reported that if the CS rate were reduced to $15 \%$, there would be worldwide cost savings of around USD2.32 billion. ${ }^{[4]}$ However, others argue that reasons for CS should be assessed to evaluate whether high rates are appropriate or not, rather than focusing on the rates themselves. ${ }^{[5]}$ Rates can be affected by the population of patients served and the expertise of the attending clinician. Auditing of events prior to CS, indications for CS and outcomes can provide insight into the appropriateness of abdominal deliveries. ${ }^{[5]}$
The Robson Ten Group Classification System (RTCGS) is a structured auditing method that has been used for monitoring CS rates in Europe. ${ }^{[5,6]}$ This ten-category classification system is based on the following obstetric concepts: category of the pregnancy; previous obstetric record; course of labour and delivery; and gestational age. The system is said to be easily reproducible and is not dependent on whether the population is at low or high risk. ${ }^{[5,6]}$ Although promoted by a Pretoria research group that has reported on the use of RTGCS in urban health facilities, ${ }^{[1,7]}$ our impression is that such auditing methods are limited in rural health facilities in SA.

\section{Objective}

To use the RTGCS to identify the leading patient categories contributing to high CS rates in a rural regional hospital.

\section{Methods}

This was a retrospective chart review of all patients who had a CS over a 3-month period at Lower Umfolozi War Memorial District Hospital, a regional hospital in northern KZN. Most of the decisions for emergency CS at the study site were made and the procedures carried out by medical officers. Specialist obstetricians performed or supervised those CSs that were expected to be surgically difficult. In general, obstetric management followed that described in the maternity care guidelines of SA. ${ }^{\left[{ }^{[}\right]}$Fetal heart rate monitoring was carried out by intermittent fetal cardiography, and labour management included the use of the partogram (labour graph). ${ }^{[8]}$ 
Data collection followed a structured format and included all relevant clinical information. Data were entered into a computer database using Microsoft Excel software. Windows SPSS version 21 was used for analysis. Results were presented as percentages, means and frequencies.

\begin{tabular}{|c|c|}
\hline Variables & \\
\hline Age (years), median, mean (SD), range & $24,25.3(6.2), 15-48$ \\
\hline Parity, mean (SD), range & $1(1), 0-7$ \\
\hline Gestational age (weeks), mean (SD), range & $37.5(3.3), 22-43$ \\
\hline \multicolumn{2}{|l|}{ HIV status } \\
\hline Infected (positive), $n(\%)$ & $377(38.4)$ \\
\hline CD4 count (cells $/ \mu \mathrm{L})$, mean (SD), range & 369.8 (198.8), 29 - 1045 \\
\hline Uninfected (negative), $n(\%)$ & $690(63.8)$ \\
\hline Result unknown or untested, $n(\%)$ & $15(1.4)$ \\
\hline $\mathrm{SD}=$ standard deviation. & \\
\hline
\end{tabular}

Institutional ethical approval to conduct the study was obtained (BE: 308/13, Biomedical Research Ethics Committee, University of KwaZulu-Natal).

\section{Results}

There were 2553 deliveries during the study period. The total number of CSs was $1085 ; 1082$ files were analysed as three files were missing, giving a CS rate of $42.4 \%$.

Table 1 shows the demographic characteristics of all patients who had a CS, and Table 2 shows RTGCs of all patients who had CSs and the percentage contribution by each group to the overall CS rate.

Table 3 shows the indications for CS per RTGC group. Group 1 was the leading group, with the combination of fetal distress, cephalopelvic disproportion, hypertensive disorders of pregnancy (HDP) and abruptio placentae being the commonest indications. Group 10 (singleton pregnancies $\leq 37$ weeks' gestational age) was the second leading group, with the additional indication of failed induction of labour.

RTGC group 5, totalling 186 patients with a scarred uterus, contributed $17.2 \%$ to the overall CS rate (Table 4 ). The majority of

Table 2. RTGC and percentage contribution by each group to the overall CS rate ${ }^{[5,6]}$

\begin{tabular}{|c|c|c|}
\hline RTGC & $n$ & $\begin{array}{l}\text { Contribution } \\
\text { to overall CS } \\
\text { rate }(\%)\end{array}$ \\
\hline Group 1. Nullipara: single cephalic term pregnancy*; spontaneous labour & 296 & 27.4 \\
\hline Group 2. Nullipara: single cephalic at term; planned CS or induced labour & 85 & 7.9 \\
\hline Group 3. Multipara without uterine scar: single cephalic at term ${ }^{*}$; spontaneous labour & 164 & 15.2 \\
\hline Group 4. Multipara without uterine scar: single cephalic term pregnancy*; planned CS or induced labour & 66 & 6.1 \\
\hline Group 5. Multipara with a scarred uterus: single cephalic term pregnancy ${ }^{\star}$ & 186 & 17.2 \\
\hline Group 6. Multipara: singleton breech presentation & 10 & 9 \\
\hline Group 7. Multipara: singleton breech presentation (including women with a scarred uterus ) & 5 & 5 \\
\hline Group 8. All multiple pregnancies (including women with a scarred uterus) & 17 & 1.6 \\
\hline Group 9. All women with single oblique or transverse pregnancy (including women with a scarred uterus ) & 0 & 0 \\
\hline Group 10. All women with a singleton cephalic preterm pregnancy $<37$ weeks' gestational age at delivery & 253 & 23.4 \\
\hline
\end{tabular}

Table 3. Indications for CS as per the RTGCS

\begin{tabular}{|c|c|c|c|c|c|c|c|c|c|}
\hline \multirow[b]{2}{*}{ RTGC group } & \multicolumn{8}{|c|}{ Indications } & \multirow[b]{2}{*}{ Total } \\
\hline & FD & CPD & Failed IOL & $\mathbf{B x}$ & HDP & Prev. CS & Abruptio & Twin & \\
\hline 1 & 155 & 130 & 0 & 1 & 6 & 0 & 4 & 0 & 296 \\
\hline 2 & 44 & 18 & 13 & 1 & 9 & 0 & 0 & 0 & 85 \\
\hline 3 & 48 & 101 & 13 & 0 & 0 & 0 & 2 & 0 & 164 \\
\hline 4 & 9 & 31 & 14 & 0 & 7 & 0 & 5 & 0 & 66 \\
\hline 5 & 0 & 0 & 0 & 0 & 0 & 186 & 0 & 0 & 186 \\
\hline 6 & 0 & 0 & 0 & 10 & 0 & 0 & 0 & 0 & 10 \\
\hline 7 & 0 & 0 & 0 & 5 & 0 & 0 & 0 & 0 & 5 \\
\hline 8 & 0 & 0 & 0 & 0 & 0 & 0 & 0 & 17 & 17 \\
\hline 9 & 0 & 0 & 0 & 0 & 0 & 0 & 0 & 0 & 0 \\
\hline 10 & 152 & 12 & 16 & 4 & 22 & 0 & 23 & 24 & 253 \\
\hline Total & 408 & 292 & 56 & 24 & 44 & 186 & 34 & 38 & 1082 \\
\hline
\end{tabular}


Table 4. Indications for CS, categorised as elective and emergency, in RTGC group 5 ( $N=186)$

\begin{tabular}{ll}
\hline Indications for CS & $\boldsymbol{n}(\%)$ \\
\hline Elective CS $(N=147)$ & \\
Prev. CS (declined VBAC) & $78(53.1)$ \\
Prev. CS $\times 2$ & $39(26.5)$ \\
Prev. CS $\times 3$ & $6(4.1)$ \\
Estimated fetal weight $>3500 \mathrm{~g}$ & $8(5.4)$ \\
HDP & $6(4.1)$ \\
Post dates & $8(5.4)$ \\
Other & $2(1.4)$ \\
Emergency CS $(N=39)$ & \\
Fetal distress & $11(28.2)$ \\
CPD & $17(43.6)$ \\
Abruptio placentae & $2(5.1)$ \\
HDP & $6(15.4)$ \\
Other & $3(7.7)$
\end{tabular}

these CSs were elective ( $n=147,79.0 \%$ ), and of these patients 53.1\% qualified for vaginal birth after their previous CS, but declined.

Emergency CSs made a very small proportion of group 5 ( $n=39$, $21.0 \%$ ). Of the emergency CSs, $43.6 \%$ were due to cephalopelvic disproportion (CPD) and $28.2 \%$ to fetal distress (Table 4).

Neonatal and perinatal outcomes are shown in Table 5. There were 1120 births; 1099 were live births, which included 1025 singletons and 74 twins. There were 21 stillbirths (1.9\%).

\section{Discussion}

Our findings show a CS rate of $42.4 \%$ over the study period. This is almost three times higher than the $15 \%$ recommended by the WHO. ${ }^{[3]}$ However, it is in keeping with the high CS rate of $40.2 \%$ reported for the whole of KZN..$^{[9]}$ Our study site was a referral centre for 17 district hospitals and local clinics, and the fact that all high-risk cases were referred to this hospital may explain the high CS rate. It is important to note that we calculated the CS rate for deliveries at the study site alone and not for the entire geographical population within the hospital's referral area.

In our study, $53.1 \%$ of women who qualified for vaginal birth after CS (VBAC) ended up having an elective CS. Offering and carrying out VBAC is one way of decreasing CS rates, especially as a number of studies have shown VBAC success rates of $\geq 50 \%$ without increasing perinatal and maternal morbidity. ${ }^{[10]}$ Our study highlights the fact that RTGC group 5 (repeat CS) contributes significantly to the high CS rates. Our impression is that insufficient counselling for VBAC occurs at the study site, meriting greater emphasis in offering VBAC and counselling all women with a previous CS to consider it if indicated.

The main contributors to the overall CS rate in our study were RTGC group 1 ( $n=296,27.4 \%)$, group $5(n=186,17.2 \%)$ and group $10(n=253,23.4 \%)$. Of note, we found a high CS rate in group 10 ( $<37$ weeks' gestation), the main indications for CS in this group being fetal distress, HDP and abruptio placentae. The high rate of CS in group 10 could reflect the many complications secondary to hypertension in our population. The indication of CPD is difficult to explain, as these pregnancies were $<37$ weeks. Further attention needs to be given to group 10 patients, for whom better decision-making may be possible at the hands of more experienced doctors and bettersupervised juniors.
Table 5. Neonatal and perinatal outcomes, $N=1120$ births

\begin{tabular}{|c|c|}
\hline Outcomes & \\
\hline \multicolumn{2}{|l|}{ Singleton pregnancies $(N=1044)$} \\
\hline Alive, $n(\%)$ & $1025(98.2)$ \\
\hline Stillbirths, $n(\%)$ & $19(1.8)$ \\
\hline $\begin{array}{l}\text { Neonatal birth weight }(\mathrm{g}) \text {, mean } \\
(\mathrm{SD}) \text {, range }\end{array}$ & 2902 (745), $1120-4980$ \\
\hline \multicolumn{2}{|l|}{ Twin pregnancies $(N=38)$} \\
\hline \multicolumn{2}{|l|}{ Alive } \\
\hline Twin 1 & 38 \\
\hline Twin 2 & 36 \\
\hline Stillbirths (fresh stillbirth, twin 2) & 2 \\
\hline \multicolumn{2}{|l|}{$\begin{array}{l}\text { Neonatal birth weight }(\mathrm{g}) \text {, mean } \\
(\mathrm{SD}) \text {, range }\end{array}$} \\
\hline Twin 1 & 2270.8 (524.2), $1140-3350$ \\
\hline Twin 2 & 2125.5 (545.7), $1000-2960$ \\
\hline $\mathrm{SD}=$ standard deviation. & \\
\hline
\end{tabular}

Studies evaluating CS using the RTGCS in LMICs are very limited. Suliman et al. ${ }^{[7]}$ reported that groups 1,3 and 5 were major contributors to CS (15.3\%, $9.3 \%$ and $83.8 \%$, respectively), accounting for $67.9 \%$ of CSs overall. These authors conducted their study in central and eastern Tshwane municipality, which is in an urban area with teaching hospitals. Group 5 was their biggest contributor to the CS rate (83.5\%), which could be explained by the high-risk nature of their patients, among whom there was an overall CS rate of $58.2 \%{ }^{[7]}$ This contrasts with our findings that group 5 contributed only $17.2 \%$ to the CS rate, of which $79 \%$ were elective CS, and among whom $53.1 \%$ qualified for VBAC but ended up with an elective CS. Only $21 \%$ of CSs in our group 5 were emergencies, of which $43.6 \%$ were due to CPD and $28.2 \%$ to fetal distress.

Recently Litorp et al., ${ }^{[1]]}$ whose study was carried out in a large teaching hospital in a major city in Tanzania, reported a CS rate of $27 \%$, with groups 1,3 and 5 contributing $12 \%, 12 \%$ and $14 \%$, respectively. ${ }^{[11]}$ All the decisions regarding CS involved specialists, in contrast to our study setting where the majority of these decisions were made by non-specialist medical officers.

Our findings are similar to those of the above studies in that RTGC groups 1 and 5 were major contributors to overall CS rates. Group 1 is amenable to corrective measures during labour. Given the fact that fetal distress and CPD were the major indications for CS in group 1, close attention needs to be given to these factors, possibly ensuring strict criteria for CS and including training on interpretation of fetal cardiotocographic recordings, and proper use and interpretation of partograms. Such measures may be expected to play a role in reducing primary CSs. Furthermore, it is known that in KZN a significant proportion of women giving birth at regional hospitals are under the age of 24 years. As many of these pregnancies are unplanned, there is a clear need to improve contraceptive services, which would result in planned pregnancies and a probable reduction in primary CS rates and subsequent repeat abdominal deliveries.

In group 10, most of the indications for CS were fetal distress, HDP and abruptio placentae. This group must be investigated further, particularly as these findings are not in keeping with those of Suliman et al. ${ }^{[7]}$ and Litorp et al. ${ }^{[11]}$ However, since these authors' studies were undertaken in urban populations, it is possible that in the rural KZN setting late booking for antenatal care, delayed referral and poor transport systems resulted in a greater number of 'emergency cases' requiring CS. 
We found that $106(9.6 \%)$ of the infants born alive were admitted to the neonatal intensive care unit (NICU), compared with the $13.9 \%$ reported by Geller et al. ${ }^{[12]}$ Prematurity complicated by birth asphyxia and transient tachypnoea of the newborn was the major indicator for admission. This is in keeping with the large number of preterm babies in group 10, which accounted for $23.4 \%$ of our overall CS rate. Although fetal distress was the leading indication for CS in our study, birth asphyxia was not the leading cause of admissions to the NICU, reflecting either timeous intervention by staff in the labour ward or unwarranted intervention due to lack of definitive diagnostic tests for fetal distress such as fetal scalp $\mathrm{pH}$ and lactate levels. A study from Tanzania revealed that many CSs were done on the basis of reliance on the CTG alone to diagnose fetal distress, without ready availability of invasive techniques such as fetal scalp pH and lactate levels to limit false-positive diagnoses. ${ }^{[13]}$ There is also a risk that the habit of easy resort to CS, especially in low-resource settings, may act as a barrier to other more effective improvements in obstetric care. ${ }^{[13]}$

Our results confirm that use of the RTGCS is feasible for auditing CS rates in a rural regional health facility and results in findings different to those in an urban setting, potentially leading to target setting relevant to the local population. This work should encourage local rural health authorities to adopt the RTGCS in an endeavour to reduce high CS rates and improve child and maternal outcomes.

\section{References}

1. Pattinson RC. Overview. In: Pattinson RC, ed. Saving Babies 2008-2009. Seventh Perinatal Care Survey of South Africa. Pretoria: Tshepesa Press, 2011.

2. Naidoo R, Moodley J. Rising rates of CSs: An audit of CSs in a specialist private practice. S Afr Fam Pract 2009;51(3):254-258. [http://dx.doi.org/10.1080/20786204.2009.10873857]

3. Villar J, Valladares E, Wojdyla D, et al. Caesarean delivery rates and pregnancy outcomes: The 2005 WHO global survey on maternal and perinatal health in Latin America. Lancet 2005;367(9525):18191829. [http://dx.doi.org/10.1016/S0140-6736(06)68704-7]

4. Gibbons JMB, Lauer JA, Betrán AP, Althabe F. The Global Numbers and Costs of Additionally Needed and Unnecessary Caesarean Sections Performed Per Year: Overuse as a Barrier to Universal Coverage. Background paper 30. Geneva: World Health Organization, 2010.

5. Robson MS, Scudamore IW, Walsh SM. Using the medical audit cycle to reduce caesarean section rates. Am J Obstet Gynecol 1996;174(1):199-205. [http://dx.doi.org/10.1016/S0002-9378(96)70394-0] 6. Robson MS. Can we reduce the caesarean section rate? Best Pract Res Clin Obstet Gynaecol 6. Robson MS. Can we reduce the caesarean section rate? Bes
2001;15(1):179-194. [http://dx.doi.org/10.1053/beog. 2000.0156]

7. Suliman S, Soma-Pillay P, McDonald AP, Pattinson RC. Factors associated with caesarean section using the Robson Ten Group Classification System. Presented at the 29th Priorities in Perinatal Care Conference, Goudini Spa, Western Cape, 9-12 March 2010.

8. National Department of Health. Maternity Care Guidelines in South Africa. 3rd ed. Pretoria: NDoH, 2007. 9. Day C, Monticelli F, Barron P, Haynes R, Smith J, Sello E. District Health Barometer 2008-2009: Health Systems Trust Technical Report. Durban: Health Systems Trust, 2010.

10. Tahseen S, Griffiths M. Vaginal birth after 2 caesarean sections (VBAC-2) - a systematic review with meta-analysis of success rate and adverse outcomes of VBAC-2 versus VBAC-1 and repeat (third) caesarean sections. BJOG 2010;117(1):5-19. [http://dx.doi.org/10.1111/j.1471-0528.2009.02351 x]

11. Litorp H, Kidanto HL, Nystrom L, Darj E, Essén B. Increasing caesarean section rates among low-risk groups: A panel study classifying deliveries according to Robson at a university hospital in Tanzania. groups: A panel study classifying deliveries according to Robson at a university hospital in Ta
BMC Pregnancy Childbirth 2013;13(107):1-10. [http://dx.doi.org/10.1186/1471-2393-13-107]

12. Geller EJ, Wu JM, Jannelli ML, Nguyen TV, Visco AG. Maternal outcomes associated with planned vaginal versus planned primary caesarean delivery. Am J Perinatol 2010;27(9):675-683. [http://dx.doi.
. org/10.1055/s-0030-1249765]

13. Maaløe N, Sorensen BL, Onesmo R, Secher NJ, Bygbjerg IC. Prolonged labour as indication for emergency caesarean section: A quality assurance analysis by criterion-based audit at two Tanzanian rural hospitals. BJOG 2012;119(5):605-613. [http://dx.doi.org/10.1111/j.1471-0528.2012.03284.x]

Accepted 2 February 2015. 